\title{
Projected ATLAS Electron and Photon Trigger Performance in Run-3
}

\author{
Fernando Monticelli, on behalf of the ATLAS Collaboration ${ }^{a, *}$ \\ ${ }^{a}$ Universidad Nacional de La Plata, IFLP, CONICET, \\ Diag 113 s/n, La Plata, Argentina \\ E-mail: Fernando.Monticelli@cern.ch
}

\begin{abstract}
ATLAS electron and photon triggers covering transverse energies from $4.5 \mathrm{GeV}$ to several $\mathrm{TeV}$ are essential to record signals for a wide variety of physics: from Standard Model processes to searches for new phenomena. During Run-3 (2021-2024) main triggers used for those physics studies will be a single-electron trigger with $\mathrm{E}_{\mathrm{T}}$ threshold around $25 \mathrm{GeV}$ and a diphoton trigger with thresholds at 25 and $35 \mathrm{GeV}$. Relying on these simple general-purpose triggers is a robust strategy that has already been tested during Run-2 (2015-2018) and avoids using a large number of analysis-specific triggers, albeit at the cost of slightly higher trigger output rates. In preparation for Run-3 data-taking, the Level-1 trigger hardware of the ATLAS calorimeter is being upgraded and the trigger software is being migrated to the multi-threaded AthenaMT framework. Impact from these modifications on the electron and photon triggers as well as their projected performance in Run-3 is presented.
\end{abstract}

40th International Conference on High Energy physics - ICHEP2020

July 28 - August 6, 2020

Prague, Czech Republic (virtual meeting)

${ }^{*}$ Speaker 


\section{Introduction}

The trigger system [1] of the ATLAS detector [2] plays a crucial role during data taking, selecting which events are saved for later use in data analysis. The trigger system has the challenging task to reduce the LHC collision rate of $40 \mathrm{MHz}$ to an average output event rate of around $1 \mathrm{kHz}$.

To achieve such goal the ATLAS trigger system has a two-level structure. The first-level (L1) trigger, implemented in custom hardware, utilises coarser-granularity signals from the calorimeters and the muon chambers to reduce the event rate from the $40 \mathrm{MHz}$ bunch crossing rate to below $100 \mathrm{kHz}$. The second stage is called the High Level Trigger (HLT) implemented in software in a dedicated computer farm which further reduces the number of events recorded to disk to an average rate of about $1 \mathrm{kHz}$ within a latency of few seconds. The HLT uses fine-granularity calorimeter information, precision measurements from the muon spectrometer and tracking information from the Inner Detector, which are not available at L1. HLT reconstruction can be executed either within the Regions of Interest (RoIs) identified at L1 or for the full detector (full-scan). At the HLT the reconstruction and identification is implemented as a sequence of fast algorithms first (to achieve a first background rejection fast) and precision algorithms after by using offline-like algorithms and techniques.

Hereafter a sequence of L1 and HLT trigger algorithms is called a "trigger" and is meant to identify one or more particles of a given type and above a given threshold of transverse energy or momentum. The configuration of the trigger is controlled by the "trigger menu", which defines a full list of L1 and HLT triggers and their configurations.

Electron and photon triggers are meant to select events with one or more electrons or photons in the detector. They are key part of the ATLAS physics programme as electrons and photons are essential components of a wide variety of physics analyses like precision Standard Model (SM) measurements [3], measurements of the properties of the Higgs boson [4] and searches for new physics beyond the SM [5].

During Run-3, the peak luminosity is expected to increase by about $10 \%$ with respect to that of Run-2 up to about $2.2 \times 10^{34} \mathrm{~cm}^{-2} \mathrm{~s}^{-1}$. It is expected that the LHC will be levelling the luminosity which means deliver peak luminosity for longer period of time making the luminosity profile very different to that of Run-2 and towards higher values, making it a more challenging scenario for the ATLAS trigger system.

\section{Triggering on electrons and photons in ATLAS during Run-2}

In the hardware-based L1 trigger, calorimeter information is used to select electron and photon candidates by verifying that the energy deposited in a given region of the Electro Magnetic (EM) calorimeter passes the $\eta$-dependent $\mathrm{E}_{\mathrm{T}}$ threshold and hadronic isolation requirements.

At the HLT, electron and photon candidates are reconstructed as clusters of energy deposited in the EM calorimeter. They are identified by making extensive use of calorimeter shower shapes and energy ratios. Tracking information is used only for electron identification at HLT. An electron is identified if a track matching the EM cluster satisfies requirements relying on the transition radiation tracker information, track-cluster matching, and tracking quantities [6]. 
The HLT fast algorithm reconstructs clusters of cells from the calorimeter within the EM RoIs identified by L1. To minimise the HLT latency, the fast algorithm uses only the second layer of the EM calorimeter to find the cell with the largest deposited transverse energy in the RoI. A cut-based selection is implemented to identify photons and a Neural-Network based algorithm called Ringer is implemented to identify electrons on triggers with threshold above $15 \mathrm{GeV}$.

The ringer algorithm exploits the property of EM showers to develop in the lateral direction in an approximately conical structure around the initial particle. This feature allows the relevant information from the calorimeters to be encoded into quantities describing energy sums $\left(\sum\right)$ of all the cells in concentric rings [6] in each calorimeter sampling layer as depicted in Fig. 1. A total of 100 rings normalized to transverse energies are fed to an ensamble of multilayer perceptron (MLP) neural networks $(\mathrm{NN})$ for each $\left(\mathrm{E}_{\mathrm{T}}, \eta\right)$ region.

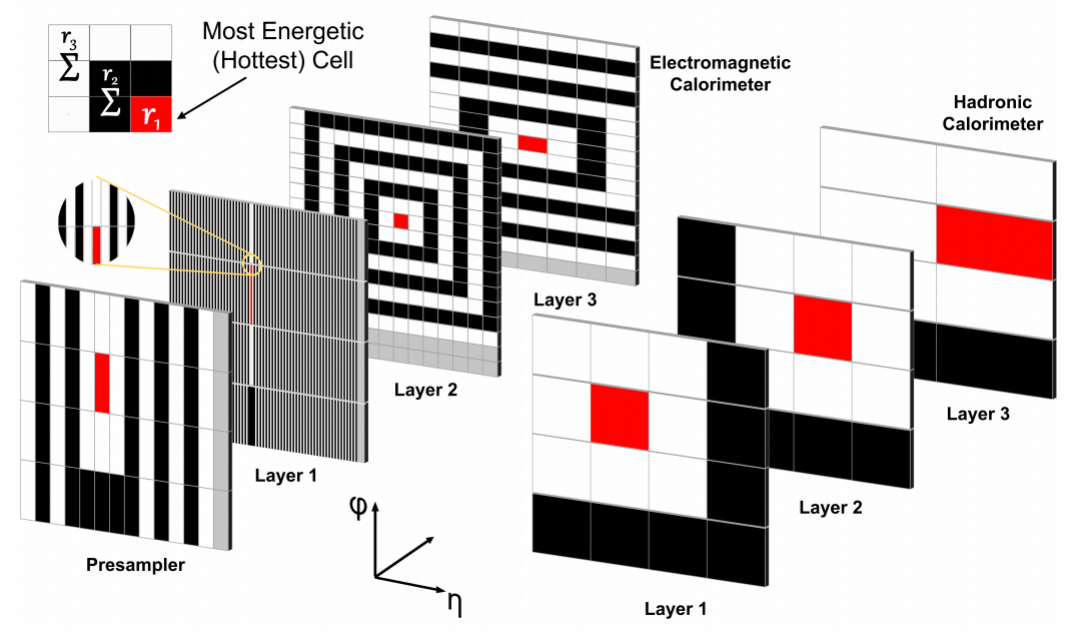

Figure 1: The concept of ring-shaped energy reconstruction in a calorimeter slice corresponding to the RoI size for $|\eta|<1.35$.

At the precision step, EM calorimeter cells within the RoI are clustered together using a slidingwindow algorithm and calibrated using a multivariate technique. Tracks reconstructed within the RoI are matched to EM clusters at the HLT to build electron candidates.

At the precision step of the HLT electron and photon identification is done as offline [7]. For electron identification, a likelihood-based approach is implemented using input from calorimeter shower-shapes, tracking, track-cluster matching and an electron probability derived from transition radiation information measured in the ATLAS Transition Radiation Tracker. During Run-2, the identification of photons was done using a cut-based selection as in Run-1 while cut values applied were reoptimized for the Run-2 conditions.

\section{Performance during Run-2}

Fig. 2 shows the efficiency of the combination of single electron triggers used during each year of Run-2 data taking with respect to offline isolated electrons satisfying offline likelihood-based tight identification. The lowest $\mathrm{E}_{\mathrm{T}}$ threshold used was $24 \mathrm{GeV}$ for 2015 and $26 \mathrm{GeV}$ for 2016-2018. 
The efficiency is computed using electrons from $Z \rightarrow e^{+} e^{-}$decay on both data and Monte Carlo (MC) simulated events. Data/MC ratios are shown in the lower canvas. The performance of the single electron triggers reaches $85 \%$ efficiency above $40 \mathrm{GeV}$ and more than $95 \%$ for $\mathrm{E}_{\mathrm{T}}>60 \mathrm{GeV}$. There is a mild drop of efficiency for high pileup but with retunes of the identification, performance was recovered in the 2017 and 2018 runs.
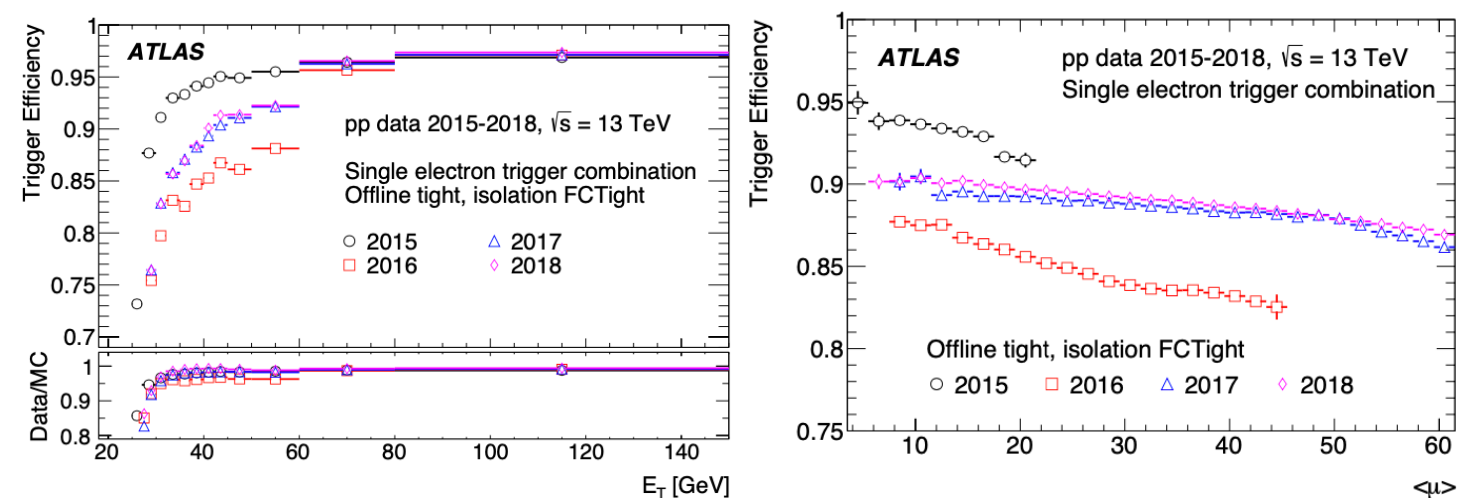

Figure 2: Efficiency of combination of unprescaled single electron triggers for 2015 (black circles), 2016 (red squares), 2017 (blue triangles) and 2018 (purple diamonds), as a function of $\mathrm{E}_{\mathrm{T}}$ (left) and as a function of average number of interactions per bunch crossing $\mu$ (right) [6].

Fig. 3 shows the efficiency of cut-based primary photon triggers with respect to offline photons. The efficiency of photon triggers is very close to $100 \%$ for photons with $\mathrm{E}_{\mathrm{T}}$ exceeding the trigger threshold by a few $\mathrm{GeV}$ for all years of Run-2 data taking and with constant performance as a function of pile-up.
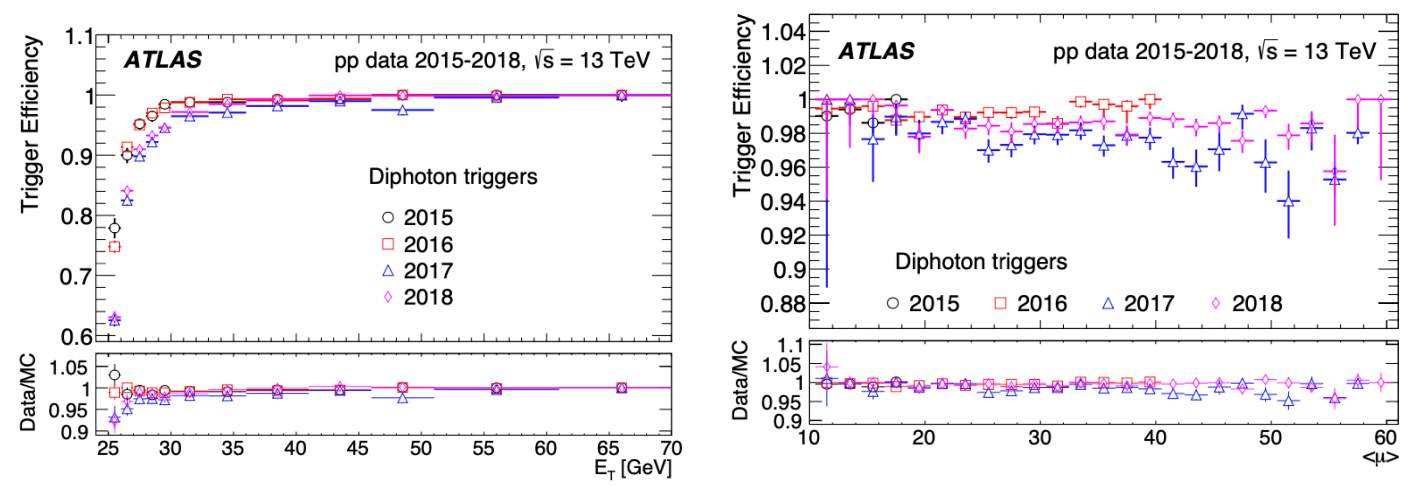

Figure 3: Efficiency of primary photon triggers requiring cut-based identification for 2015 (black circles), 2016 (red squares), 2017 (blue triangles) and 2018 (purple diamonds), as a function of $\mathrm{E}_{\mathrm{T}}$ (left) and as a function of average number of interactions per bunch crossing $\mu$ (right) [6].

\section{Improvements towards Run-3}

For Run-3 the ATLAS Liquid Argon calorimeter readout is upgraded to increase tenfold the longitudinal and transverse granularity of the calorimeter information provided to the L1 trigger system. Instead of a single trigger tower which combines all four EM calorimeter layers, they are 
read out separately, and in Layer-1 and Layer- 2 of the EM calorimeter the granularity is increased four times in $\eta$. This new structure is called Supercell. Also a new electron Feature EXtractor (eFEX) processor with different reconstruction algorithms will be installed. These algorithms use a $0.3 \times 0.3$ window in $\eta \times \phi$, and select the local maximum of energy only in the Layer- 2 of the EM calorimeter. Then, the most energetic neighboring cell in $\phi$ is selected, and with this, the cluster with $0.3 \times 0.2$ cells in fine layers, and $0.1 \times 0.2$ in coarse layers is built.

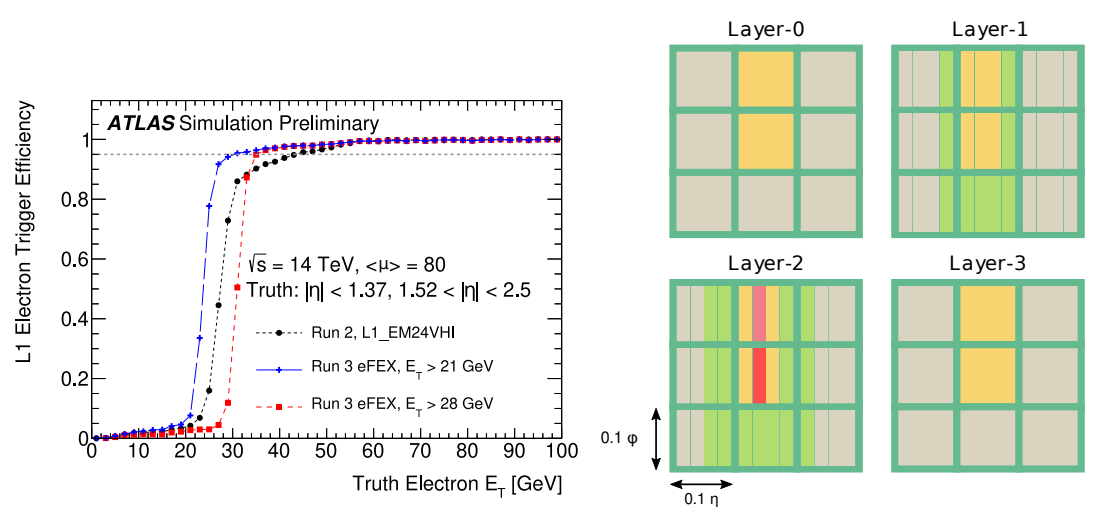

Figure 4: Left: L1 trigger efficiency with respect to truth electrons as a function of their transverse energy. Black and blue curves have the same rate but different thresholds, and use different algorithms. The red curve has half of the rate of the black curve [8]. Right: schematic diagram of how supercells are grouped.

A schematic display of this grouping procedure is shown in Fig. 4 (right). The finer granularity of the Supercells enables more sophisticated rejection of jet backgrounds than in Run-2 through the use of shower shape variables [9]. Similarly to Run-2, this additional selection is unnecessary for the L1 EM objects above $60 \mathrm{GeV}$. Fig. 4 (left) shows that a Run-3 trigger tuned to the same rate as a Run-2 trigger can have a much lower $\mathrm{E}_{\mathrm{T}}$ requirement, while if the $\mathrm{E}_{\mathrm{T}}$ requirements for Run-2 and Run-3 triggers are similar, the expected rate of the Run-3 trigger reduces to a half.

At the HLT there are improvements being implemented for Run-3 data taking. ATLAS is moving towards a new multi-thread version of the software framework ATHENA [10]. This will improve the CPU resource usage of the HLT computer farm.

At the fast reconstruction step the ringer algorithm will be implemented and used as default for all electron triggers and not just for those with $\mathrm{E}_{\mathrm{T}}>15 \mathrm{GeV}$ as in Run-2. This will increase the early background rejection for low $\mathrm{E}_{\mathrm{T}}$ and improve the CPU usage at the HLT. The usage of ringer algorithm on photon triggers is also being evaluated.

At the precision step of the HLT in Run-3 electrons and photons will be reconstructed using same algorithms used offline. Instead of Sliding-window clustering algorithm, superclusters will be used. With this algorithm, dynamically aggregated cells with energy above noise threshold (called topological clusters or topo-clusters) are grouped to additional nearby satellite topo-clusters to take into account for bremsstrahlung radiation [7]. In addition a Gaussian sum filter (GSF) algorithm, a non-linear generalization of the Kalman filter [7], will be used to refit tracks candidates at HLT for electron triggers. This will improve the online-offline energy resolution with respect to Run-2. 


\section{Conclusions}

Electron and photon triggers in ATLAS are a key part of the physics programme as they are the first and unavoidable selection of events with electrons and/or photons in the final state. During Run-2 the performance of the electron and photon triggers fulfilled the ATLAS physics analysis requirements. For the Run-3 of the LHC, ATLAS electron and photon trigger reconstruction and selection will benefit from the improvements presented in this document and are expected to achieve a performance superior to that of Run-2.

\section{References}

[1] ATLAS Collaboration. Performance of the ATLAS Trigger System in 2015. Eur. Phys. J. C, 77(5):317, 2017.

[2] ATLAS Collaboration. The ATLAS experiment at the CERN large hadron collider. 2008 JINST 3, S08003.

[3] ATLAS Collaboration. Measurement of $W^{ \pm} Z$ production cross sections and gauge boson polarisation in $p p$ collisions at $\sqrt{s}=13 \mathrm{TeV}$ with the ATLAS detector. Eur. Phys. J. C, 79(6):535, 2019.

[4] ATLAS Collaboration. Constraints on off-shell Higgs boson production and the Higgs boson total width in $Z Z \rightarrow 4 \ell$ and $Z Z \rightarrow 2 \ell 2 v$ final states with the ATLAS detector. Phys. Lett. $B$, 786:223-244, 2018.

[5] ATLAS Collaboration. Constraints on mediator-based dark matter and scalar dark energy models using $\sqrt{s}=13 \mathrm{TeV} p p$ collision data collected by the ATLAS detector. JHEP, 05:142, 2019.

[6] ATLAS Collaboration. Performance of electron and photon triggers in ATLAS during LHC Run 2. Eur. Phys. J. C 80 (2020) 47.

[7] ATLAS Collaboration. Electron and photon performance measurements with the ATLAS detector using the 2015-2017 LHC proton-proton collision data. 2019 JINST 14, P12006.

[8] ATLAS Collaboration. Level-1 Calorimeter Trigger Public Results, https://twiki.cern.ch/twiki/bin/view/AtlasPublic/L1CaloTriggerPublicResults, 2018.

[9] ATLAS Collaboration. Technical Design Report for the Phase-I Upgrade of the ATLAS TDAQ System. Technical Report CERN-LHCC-2013-018. ATLAS-TDR-023, Sep 2013.

[10] ATLAS Collaboration. AthenaMT: upgrading the ATLAS software framework for the manycore world with multi-threading. J. Phys. Conf. Ser. 898042009. 